\title{
Simulations of Thermal Processing of Volatiles on Carbonaceous Asteroids
}

\author{
PIERRE HAENECOUR AND THOMAS ZEGA
}

The University of Arizona

Presenting Author: haenecour@arizona.edu

Small bodies such as asteroids give us the opportunity to study the original materials that formed the planets in the protoplanetary disk. They contain bio-essential components, e.g., organics and water-bearing phases, that we think contributed to the ingredients of life and water on Earth. Laboratory analysis of meteorites shows that these components are intimately mixed at the nanoscale [1].

To constrain whether asteroids delivered bio-ingredients to the Eartrh, it is important to retrace their origins and evolution histories. The response of fine-grained materials in carbonaceous chondrites to secondary alteration is important for understanding active processes affecting volatiles on the surfaces of and within the chondrite-parent asteroids including melting, volatile loss, elemental diffusion between grains, and driving hydrothermal processing [1]. The presence of hydrous minerals in many carbonaceous chondrites provides evidence of low-temperature hydrothermal on asteroids [2]. Constraining the effects of these different thermal processes is critical to understanding past and ongoing surface processing of asteroids.

We previously reported on in-situ flash- and step-heating experiments of matrix material of the Murchison (CM2), Tagish Lake (C2-ung.) and Acfer 094 (C2-ung.) chondrites inside a Hitachi SU9000 $30 \mathrm{keV}$ scanning transmission electron microscope (STEM). Our results showed significant changes to their microstructure and elemental compositions, such as melting and formation of $\mathrm{Fe}-\mathrm{Ni}$ nanoparticles, occurred only after heating above $500^{\circ} \mathrm{C}[3]$. Here we report results from new in-situ heating experiments of fine-grained materials from the Chwichiya 002 (C3.00-ungrouped) and Tarda (C2-ungrouped) carbonaceous chondrites. Matrix materials of the three meteorites were crushed and then deposited onto Norcada heating chips. Each sample was heated in situ using the Hitachi Blaze heating holder in a Hitachi HF5000 TEM/STEM at the University of Arizona (UA). The in situ heating experiments were carried out in vacuum $\left(<10^{-5} \mathrm{~Pa}\right)$ at temperatures up to $1075^{\circ} \mathrm{C}$. We will describe the results at the meeting.

References. [1] Le Guillou et al. (2014) GCA 131, 368-392. [2] Wadhwa et al. (2020) Annu. Rev. Earth Planet. Sci. 48: 23358. [3] Brearley (2014). Treatise on Geochemistry (2nd Ed), Vol. 1, 309-334. [4] Haenecour et al. (2019). Asteroid Science Workshop, abstract \#2046. 